\title{
Summary of the Structure Functions and Low-x working group
}

\author{
Joanne E. Cole*, Jianwei Qiu ${ }^{\dagger}$ and Un-ki Yang** \\ ${ }^{*}$ H.H. Wills Physics Laboratory, University of Bristol, Bristol, BS8 1TL, UK \\ ${ }^{\dagger}$ Department of Physics and Astronomy Iowa State University, Iowa 50011, USA \\ ${ }^{* *}$ Enrico Fermi Institute, University of Chicago, Chicago, Illinois 60637, USA
}

\begin{abstract}
We report a summary of the structure function working group which covers a wide range of the recent results from HERA, Tevatron, RHIC, and JLab experiments, and many theoretical issues from low $x$ to high $x$.
\end{abstract}

Keywords: structure functions, low- $\mathrm{x}$

PACS: 12.38.-t,12.38.Mh, 12.38.Qk,13.60.Hb

\section{INTRODUCTION}

Much of the predictive power of Quantum Chromodynamics (QCD) is provided by universality of the non-perturbative functions, in particular, the parton distribution functions (PDFs), in factorization theorems for hard processes. With the aid of factorization and perturbative calculation of short-distance dynamics, the universality allows us to extract a set of PDFs from some reactions and then use them to predict observables in other reactions. Knowledge of PDFs is critical for testing QCD dynamics in asymptotic region at existing facilities, as well as, for making predictions for future facilities, like the Large Hadron Collider (LHC). It is also essential for exploring non-perturbative QCD dynamics when the extracted PDFs are compared with what calculated in lattice QCD or in effective field theory approaches.

With only one identified hadron, structure functions of inclusive lepton-hadron deeply inelastic scattering (DIS) are clean observables for extracting PDFs. After more than 30 years of continuous effort, and many generations of machines and detectors, measurements of proton structure functions have become the benchmark tests of QCD dynamics. With the HERA at DESY, we are able to explore the kinematic region with the Bjorken $x_{B}$ as low as $10^{-5}$ while staying the DIS regime. The continuous growth of structure functions as $x_{B}$ decreases raises an urgent question: when such growth will hit the unitarity limit and slow down? The knowledge of structure functions and low $x$ physics is extremely important for testing QCD dynamics and our ability to explore new physics beyond the standard model.

Our working group had a total of 46 talks divided into 9 sessions including one joint session with Electroweak and Beyond the Standard Model working group. In this writeup, we summarize the recent achievements, progresses, and open questions that were presented at our working group meetings. We organize this summary into seven 
parts:

1. Structure function measurements at low $x$

2. Structure functions and PDFs at high $x$

3. Progress in the determination of PDFs

4. Toward QCD precision tests

5. Low- $x$ physics: parton evolution and saturation

6. Nuclear structure functions and nuclear PDFs

7. New approaches to PDFs

\section{STRUCTURE FUNCTION MEASUREMENTS AT LOW $X$}

Measurements of the proton structure function, $F_{2}$, in neutral current (NC) deep inelastic scattering (DIS) at HERA are vital for testing the predictions of perturbative QCD and in the determination of the parton distribution functions of the proton. Recent results from the two general-purpose detectors, $\mathrm{H} 1$ and ZEUS, cover five orders of magnitude in the photon virtually, $Q^{2}$, and in the Bjorken scaling variable, $x[1]$.

The possibility afforded by HERA of studying the structure functions down to values of $x$ as low as $\sim 10^{-6}$ is important, as it gives access to partons which have undergone a large number of QCD branching processes. The density of these partons, both gluons and the so-called "sea" quarks has be found to increase dramatically as $x$ decreases, which may indicate the need to taken into account non-linear effects in QCD evolution, such as saturation.

The double-differential cross section for inclusive NC DIS is given by:

$$
\frac{x Q^{4}}{2 \pi \alpha^{2} Y_{+}} \frac{d^{2} \sigma}{d x d Q^{2}}=\sigma_{r}=F_{2}\left(x, Q^{2}\right)-\frac{y^{2}}{Y_{+}} F_{L}\left(x, Q^{2}\right)-\frac{Y_{-}}{Y_{+}} x F_{3}\left(x, Q^{2}\right)
$$

where $Y_{ \pm}=1 \pm(1-y)^{2}$, in which $y=Q^{2} / x s$ is the inelasticity, $s$ is the total squared center-of-mass energy and $F_{2}, F_{L}$ and $F_{3}$ are the structure functions of the proton. The quantity, $\sigma_{r}$ is also defined in this equation, which is known as the reduced cross section.

Although the precision measurements of $F_{2}$ exist over such a large kinematic range, the same cannot be said for the longitudinal structure function, $F_{L}$, which has not been directly measured at HERA. $F_{L}$ is directly sensitive to scaling violations and hence to the gluon content of the proton and is therefore crucial to our understanding of proton structure.

The final term in equation (11) contains $x F_{3}$, or the parity violating structure function. This structure function is, however, only important at high $Q^{2}$ and will not be considered further in this section, where the interest is primarily in low $Q^{2}$ structure function measurements.

The rise of $F_{2}$ with decreasing $x$ persists down to very low values of $Q^{2}$ [2], although it is known that as $Q^{2} \rightarrow 0, F_{2} \rightarrow$ constant $Q^{2}$, as it must in order to satisfy the conservation of the electromagnetic current. It is also known that around $Q^{2} \sim 1 \mathrm{GeV}^{2}$, perturbative QCD begins to breakdown and phenomenological models must be invoked to explain the behavior of the $F_{2}$ data. 


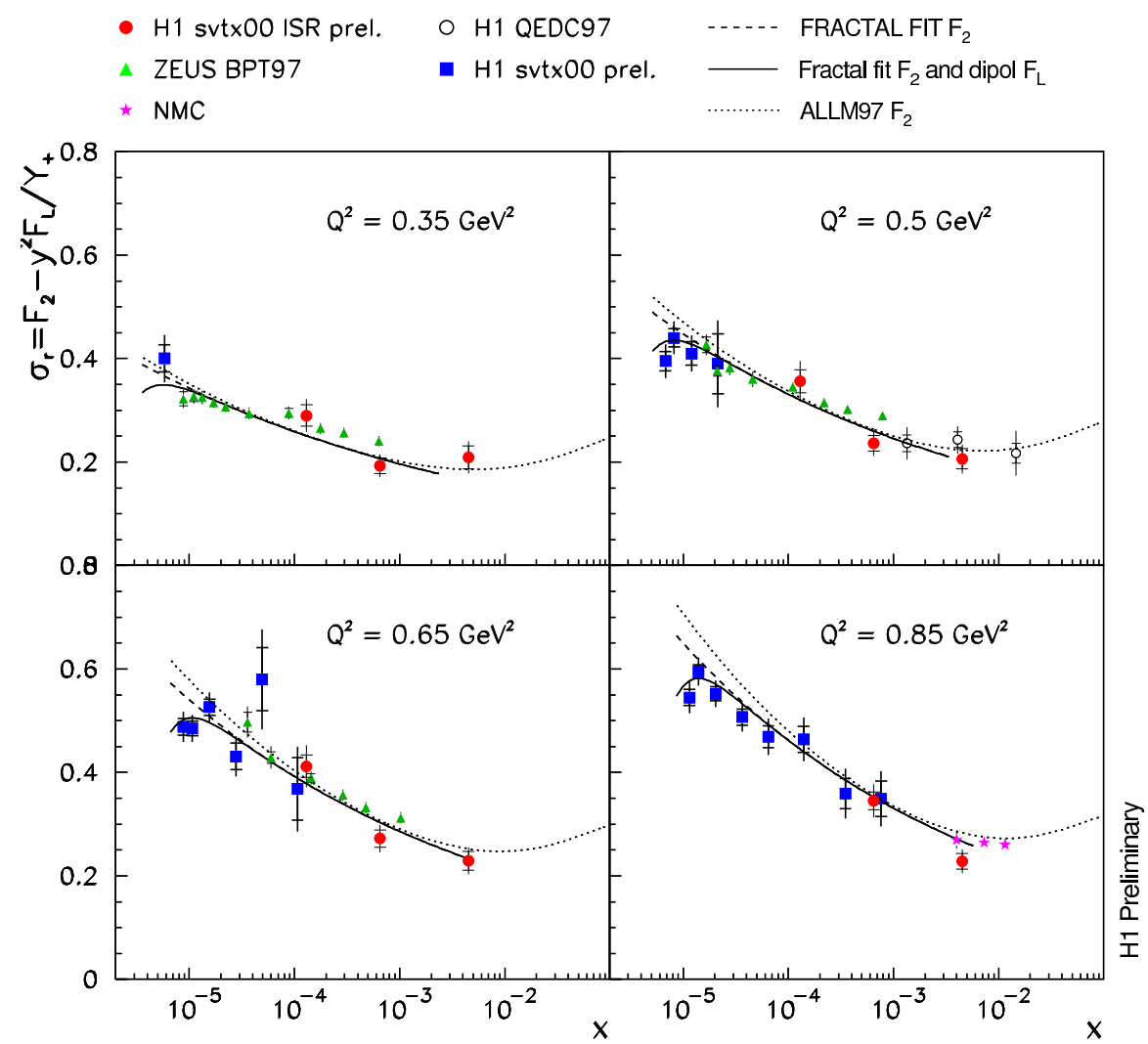

FIGURE 1. Compilation of reduced cross section measurements for $Q^{2}<1 \mathrm{GeV}^{2}$ from the H1, ZEUS and NMC Collaborations.

From an experimental point of view, accessing very low values of $Q^{2}$ is technically challenging, but has been achieved by the HERA experiments using a number of different techniques. The H1 Collaboration presented recent measurements of the reduced cross section at low $Q^{2}$ using two of these techniques, namely, via the identification of QED Compton events [3] and using a small sample of data in which the interaction vertex was intentionally shifted by $+70 \mathrm{~cm}$ toward the outgoing proton beam direction [4], effectively extending the acceptance of the $\mathrm{H} 1$ detector to values of $Q^{2}$ down as low as $0.35 \mathrm{GeV}^{2}$. Using this so-called "shifted vertex" data sample, they have also specifically identified events in which an energetic photon was emitted by the incoming lepton prior to its interaction with the proton; these initial-state radiative (ISR) events give access not only to even lower values of $Q^{2}$, but also to higher values of $x$, giving a wide coverage in $x$ at low $Q^{2}$.

These measurements are shown in figure 1, in which it can be seen that $F_{2}$, and hence the reduced cross section, rises with decreasing $x$, even at low values of $Q^{2}$. The only exception to this behavior is at the very lowest values of $x$, at which the contribution to the reduced cross section from the longitudinal structure function, $F_{L}$, becomes significant, causing $\sigma_{r}$ to decrease. As can be seen in equation (1), the contribution to the reduced cross section from $F_{L}$ is suppressed for all but the highest values of $y$ (low $x$ ). This be- 


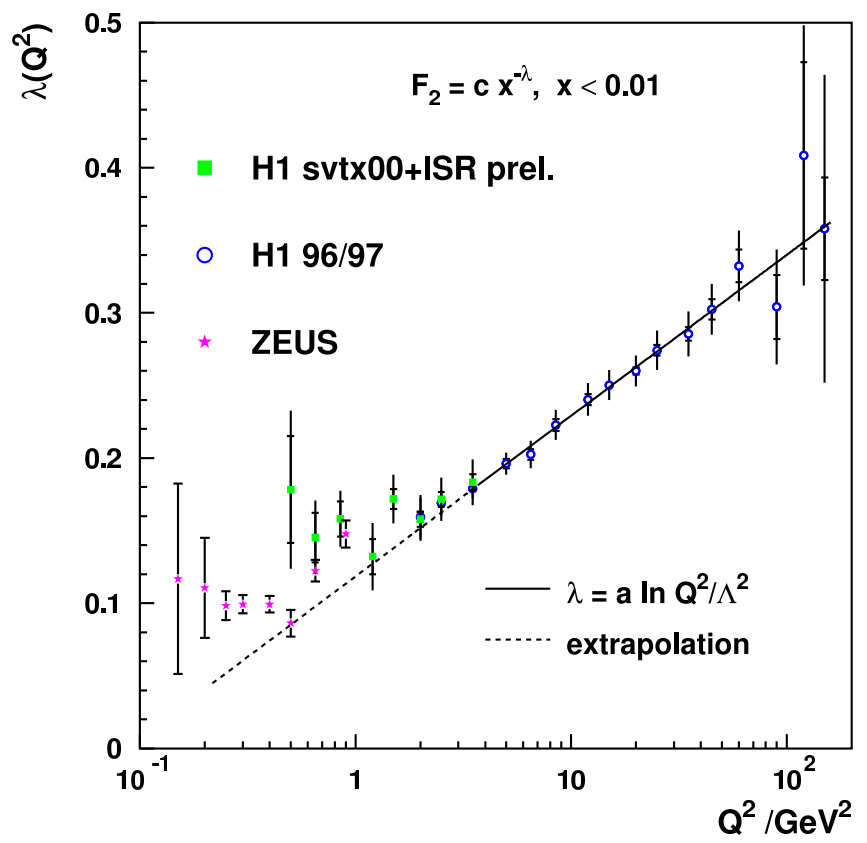

FIGURE 2. Compilation of selected HERA results on the parameter $\lambda$, obtained from fits of the form $F_{2}=c\left(Q^{2}\right) \cdot x^{-\lambda\left(Q^{2}\right)}$ to low $x$ data

havior can be exploited to perform an extraction of $F_{L}$, albeit in a model-dependent way. The resulting $F_{L}$ points were also shown at this workshop [4] and are already able to discriminate between different PDF parameterizations.

The low $Q^{2} F_{2}$ data can be fitted in order to quantify the change of the low $x$ slope of $F_{2}$ with $Q^{2}$. Figure 2 shows the result of just such a fit, performed for $x<0.01$ by the H1 Collaboration. The expected change in behavior around $Q^{2} \sim 1 \mathrm{GeV}^{2}$, is clearly observed.

\section{STRUCTURE FUNCTIONS AND PDFS AT HIGH $X$}

Structure Functions at high $x$ region has brought many attentions at this workshop. Recently, it has been realized that it is very important to understand this region in order to achieve precise electroweak measurements and to extract new physics signals from HERA, Tevatron and LHC at high $Q^{2}$ region. A large uncertainty on the PDFs at very high $x$ and low $Q^{2}$ region can make a big impact on the high $Q^{2}$ region even at intermediate $x$ due to the effect of DGLAP evolution.

Most precise data on high $x$ come from the traditional fixed target experiments (SLAC/BCDMS/NMC). But their high $x$ data corresponds to low $Q^{2}$ region where we face many challenges in understanding all non-perturbative QCD and nuclear effects. One clean way is to probe the structure functions at high $x$ and $Q^{2}$ directly. Both $\mathrm{H} 1$ and ZEUS [5] showed measurements of the cross sections for neutral and charged-current scattering as a function of $Q^{2}$ using polarized beams. The measured cross sections are 


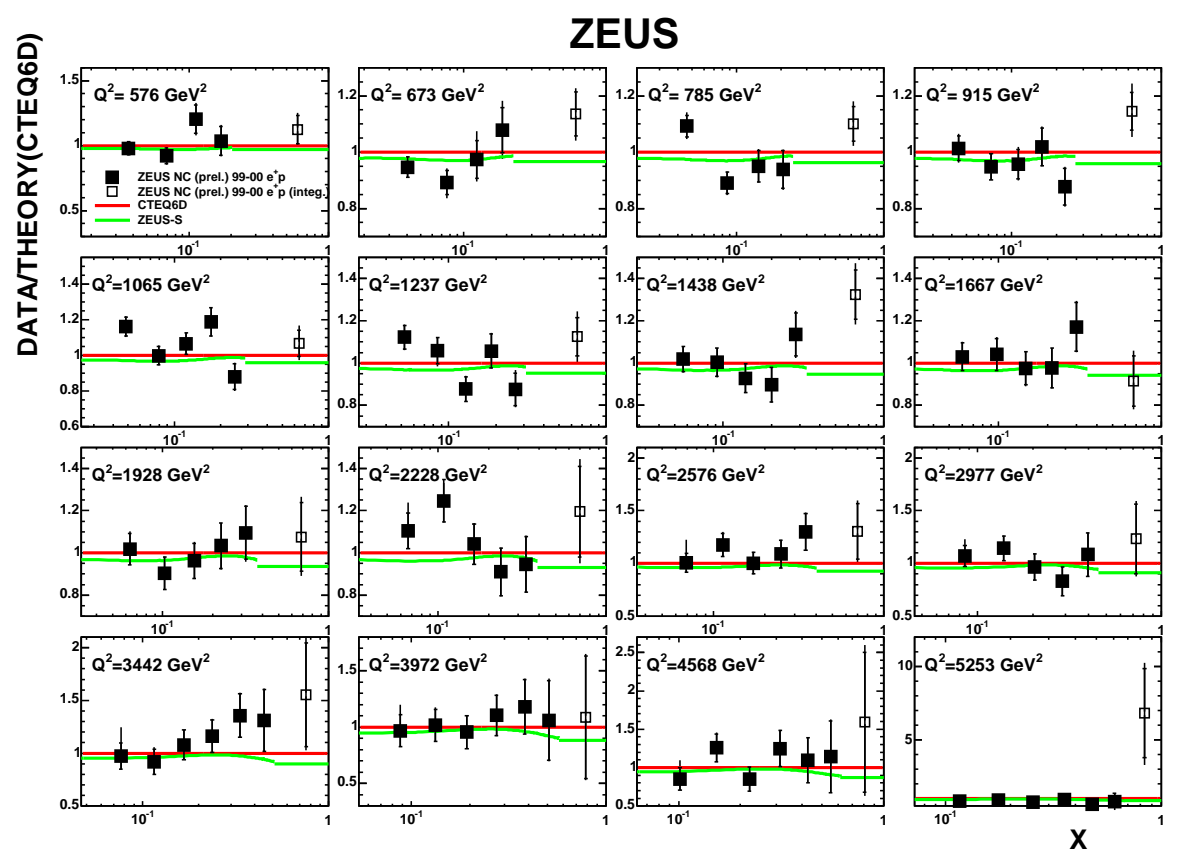

FIGURE 3. [Left] The ratio of the ZEUS differential cross sections data and predictions using CTEQ6D PDFs.

well described by the Standard Model. But more data is required to constrain parton distributions functions at high $x$. The ZEUS [6] presented a very promising method to probe the PDFs up to $x=1 \mathrm{using}$ the jet information $\left(E_{\text {jet }}\right.$ and $\left.\theta_{\text {jet }}\right)$ to calculate the value of $x$. Events with no jets reconstructed within their fiducial volume is assumed to come from very high $x_{e d g e}$ to 1 . The measured cross sections using early dataset show good agreements with the predictions using CTEQ6D PDFs, shown in Figure 3. However, their highest $x$ data tend to be higher than the predictions. Thus, it would be interesting to see their results using a full dataset.

The NuTeV [7] presented their final differential cross sections using neutrino-iron scattering. The extracted $F_{2}$ and $x F_{3}$ from their differential cross sections are $20 \%$ higher than the CCFR measurements, and $10-15 \%$ higher than the BCDMS $F_{2}$, as shown in Figure 4. They explained that two third of the difference between the NuTeV and CCFR measurements is due to an improved calibration of the magnetic field, and a better modelling in Monte Carlo. This result implies that that nuclear effect in neutrino scattering is different from that in the charged lepton scattering at high $x$. Thus, we need to resolve this difference before the $\mathrm{NuTeV}$ data can be used in a global PDFs analysis to constrain the PDFs at high $x$. It would be interesting to see their QCD fit results. A possible difference in the nuclear effect can be resolved by the CHORUS data on the lead target, and future Minerva/MINOS results.

The ratio of $d$ and $u$ quarks at high $x$ primary comes from the measurements of $F_{2}$ (deuterium) and $F_{2}$ (proton). Because of a large uncertainty of nuclear binding effect on deuterium target, this ratio is poorly known. Figure [5]left] shows the NMC $F_{2}^{d} / F_{2}^{p}$ with and without nuclear correction [8]. With a nuclear correction, the NMC data favors 

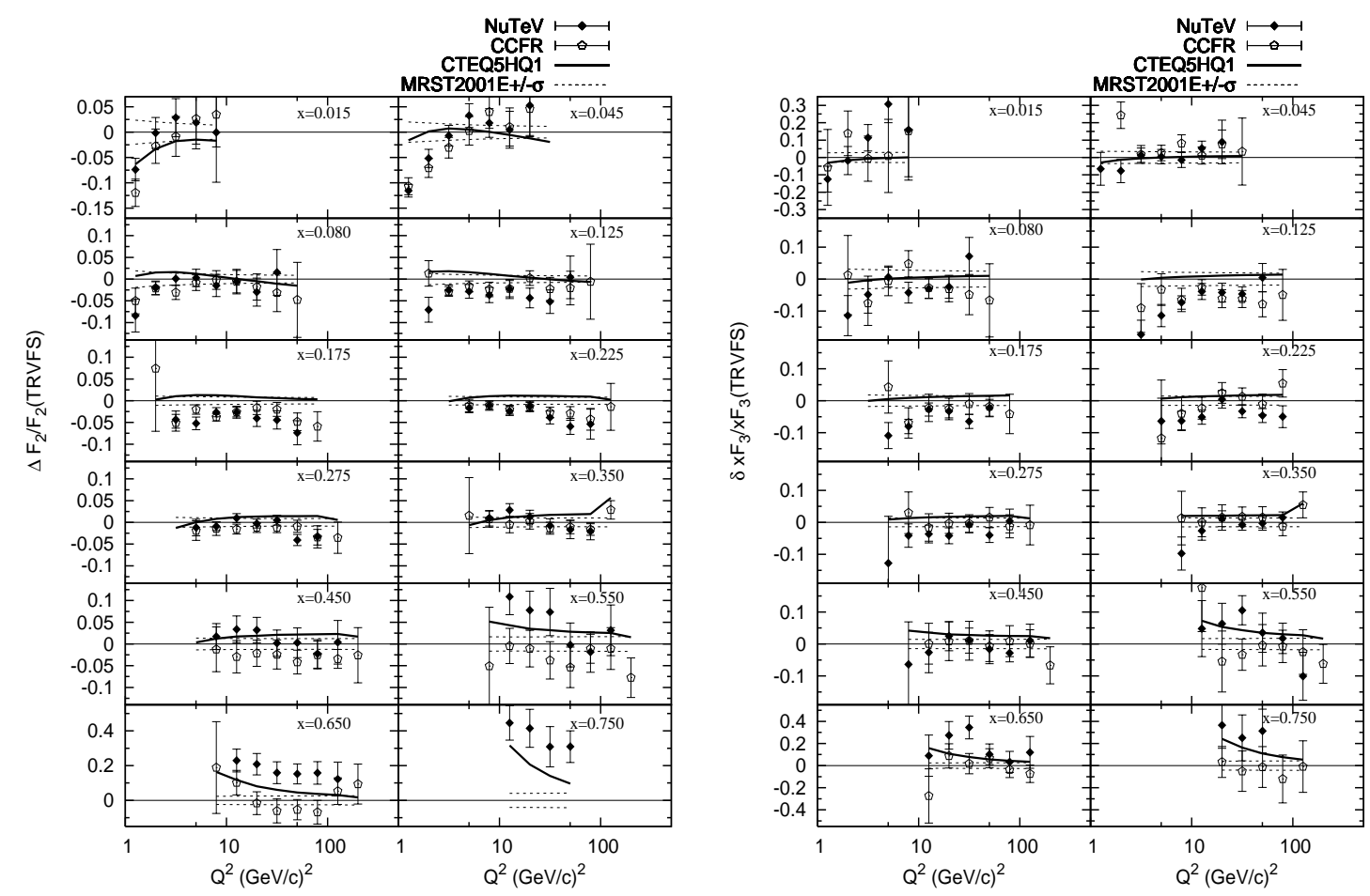

FIGURE 4. NuTeV $F_{2}\left[\right.$ left] and $x F_{3}$ [right] data are compared with CCFR data and the NLO prediction with MRST2001E.

0.2 for $d / u$ as $x \rightarrow 1$, which is of theoretical interest for nuclear physics community. However, the size of nuclear binding correction is still controversial. S.Kuhn [9] presented dedicated JLab efforts to study $d / u$ at high $x$. Their programs are to study the effect of nuclear binding on neutron structure, and to measure the structure functions of a free neutron by detecting a slow spectator proton. Information on $d / u$ can be also extracted from $W$ production data at the Tevatron. The CDF collaboration [10] measured the forward-backward charge asymmetry of electrons from $W$ boson decays. In order to get a better $d / u$ sensitivity on higher $x$ region, they have looked at a higher electron $E_{T}$ region. Fig 5 right] shows comparisons with the NLO RESBOS predictions using CTEQ6M and MRST 2001 PDFs. At high $\eta$, the CDF data tends to favor higher $d / u$ value at high $x$. Thus, it would be interesting to compare with the PDFs which was extracted, assuming a large nuclear correction on the deuterium target. They expect to have a big improvement on this measurement by reconstructing $W$ rapidity directly.

At the workshop, one of the hot subjects was the phenomenon of a parton-hadron duality which states that the average behavior of the nucleon resonances follows the DIS scaling limit curve. JLab has very precise data at high $x$ and low $Q^{2}$ region (where a resonance production occurs). Besides many theoretical issues discussed by S. Liuti [11], C. Keppel and I. Niculescu [12] demonstrated that the duality holds for $F_{2}$ proton, EMC 

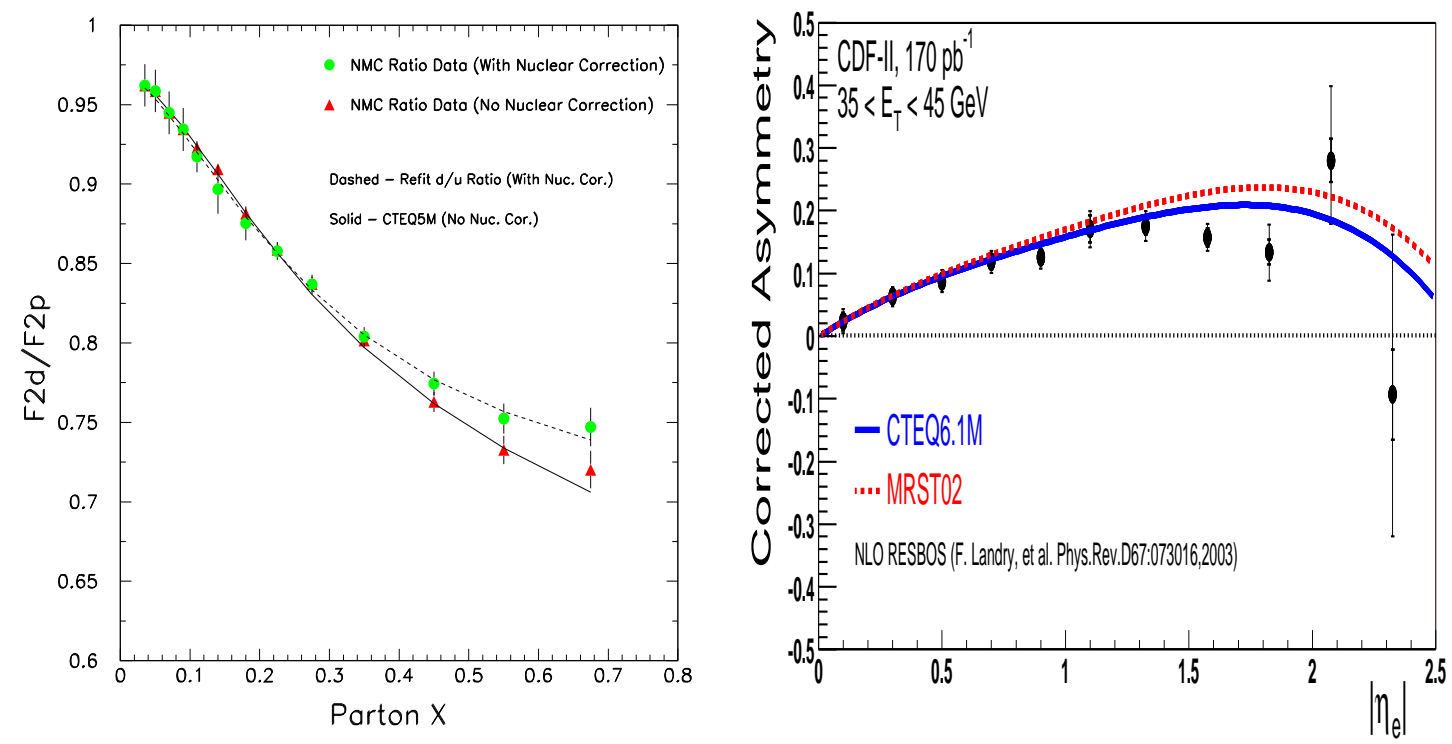

FIGURE 5. [Left] The ratio of $F_{2}$ data on the deuterium and hydrogen targets with and without the nuclear corrections. [Right] The CDF lepton charge asymmetry is compared with predictions with CTEQ6M and MRST02 PDFs using a NLO RESBOS calculation.

effect, and even spin structure functions in the region of $Q^{2}>0.5 \mathrm{GeV}^{2}$. Issues are whether non-perturbative power corrections between DIS and resonance region is same, and DGLAP evolution \& factorization works in the resonance region too. S. Liuti's studies suggest that the size of the higher twist effect may not be same. However, A. Bodek [13] showed that all DIS $F_{2}$ data and JLab's resonance data are well described by his Bodek-Yang leading-order model, as shown in Figure 6 . This implies that there is not much difference in the power corrections between two regions. This model uses a new scaling variable $\xi_{w}$ and $Q^{2}$ dependent $K$ factors to all PDFs to describe both pQCD and non-perturbative $\mathrm{QCD}$ regions very smoothly.

A community of neutrino oscillation physics have started to pay attention on this non-perturbative QCD region. For precise measurements of mass splitting and mixing angles, neutrino oscillation experiments (MINOS, NOvA, and T2K) need to have a good understanding of neutrino cross section at low energy. This point was well presented by H. Gallagher [14]. Certainly this would be a place where DIS, nuclear physics, and neutrino physics communities need to make a coherent effort.

At the end of the workshop, PDF uncertainties at the Tevatron and impact on various measurements are presented by F. Chlebana, and A. Harel [15]. Chelbana discussed many ideas to constrain the PDFs using the Tevatron data. Figure 7 shows the latest status of the Tevatron jet data with the NLO predictions where there is no observed discrepancy at high $E_{T}$ region. These measurements are currently dominated by the jet energy scale uncertainties. In future, it would be important to separate PDF effects from any new physics signal. F. Chlebana He also pointed out that it is crucial to measure the size of heavy flavor quarks densities for Higgs and Top physics. 

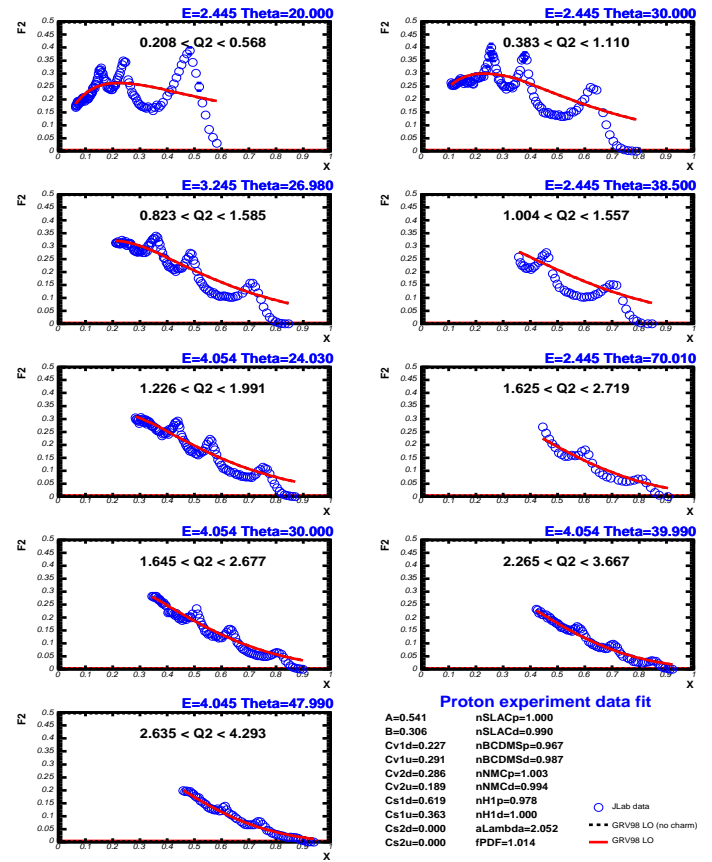

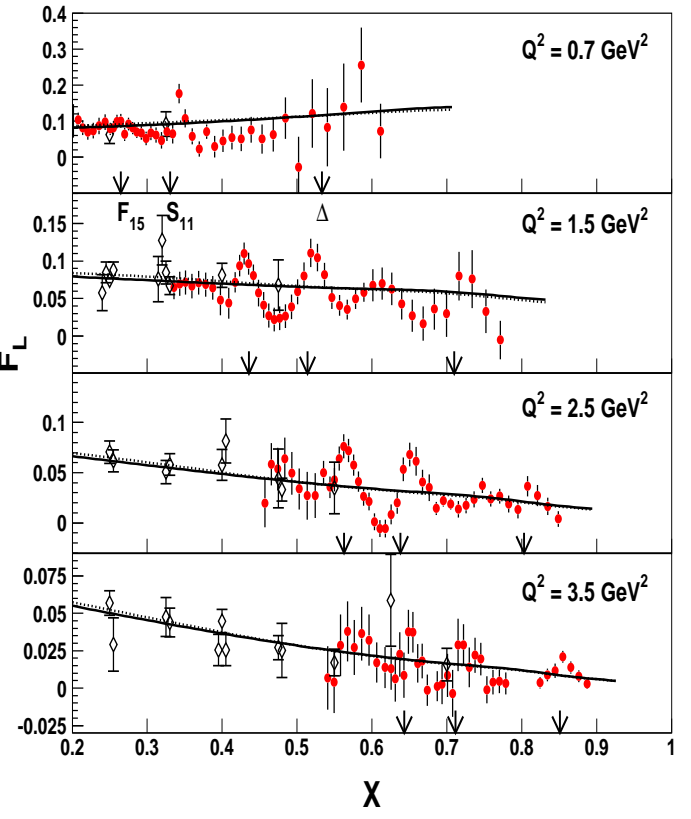

FIGURE 6. Comparisons of the predictions of Bodek-Yang model to resonance electro-production data on proton: JLab $F_{2}$ proton [left], and $F_{L}$ proton[right].
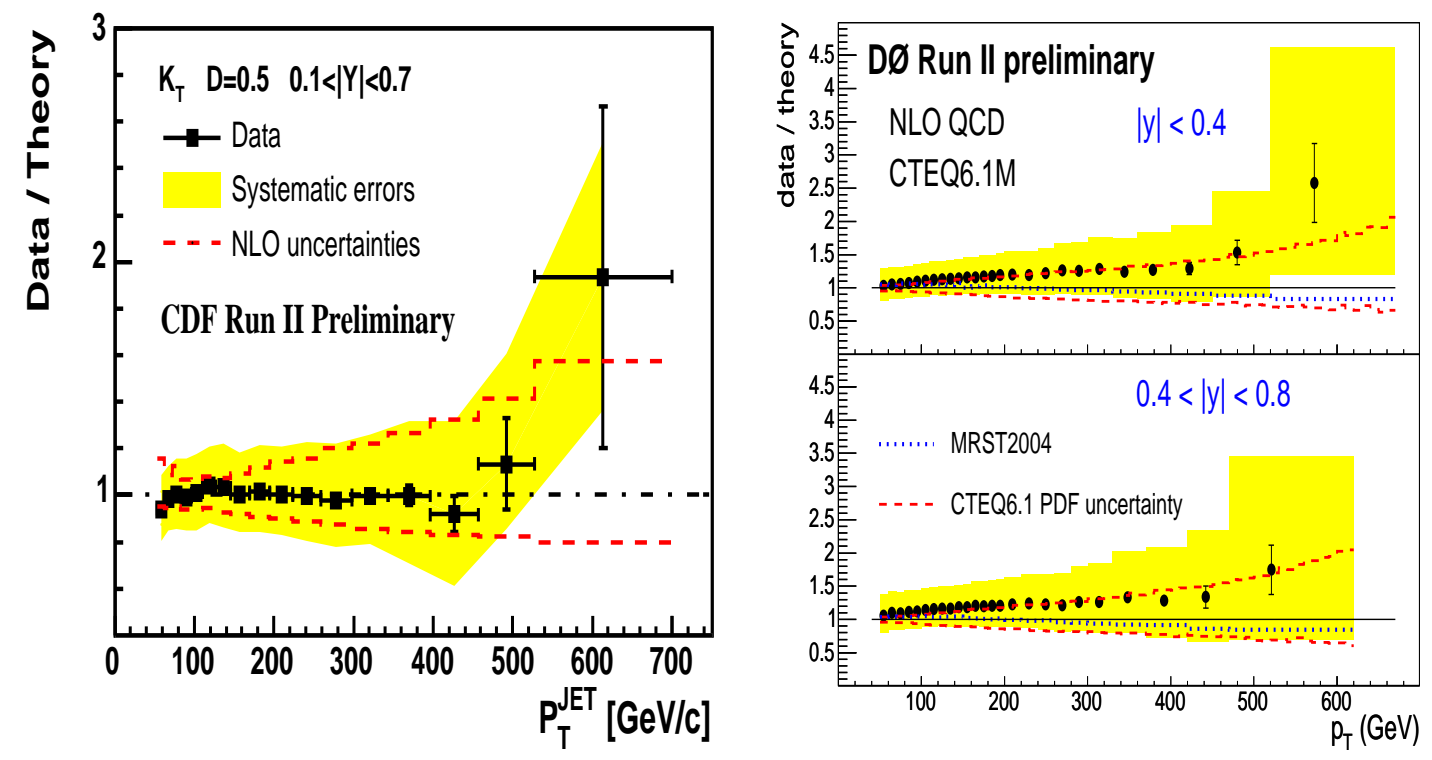

FIGURE 7. The ratios of the inclusive jet cross section data and the NLO predictions: CDF data using $K_{T}$ algorithm [left] and D0 data using cone algorithm[right]. 


\section{PROGRESS IN THE DETERMINATION OF PARTON DISTRIBUTION FUNCTIONS}

The structure functions discussed in the previous section can be expressed in terms of the parton distribution functions (PDFs) of the proton. The structure function, $F_{2}\left(x, Q^{2}\right)$ is proportional to the sum of the quark and antiquark PDFs. At low $x, F_{2}$ is therefore sensitive to the sea quark distributions and hence is indirectly sensitive to the gluon density. The longitudinal structure function, $F_{L}$, is directly sensitive to the gluon density. The parity-violating structure function, $x F_{3}\left(x, Q^{2}\right)$, is proportional to the difference between the quark and antiquark PDFs, making it sensitive to the valence quark distributions.

The PDFs of the proton may be extracted by fitting, among other things, the HERA structure function data. These fits have been performed by a number of different groups, as well as the experimental collaborations themselves. It is crucial that the best possible understanding of the proton PDFs is achieved, given their central role in predictions for other processes, for example, at the LHC.

The traditional method of determining the PDFs of the proton relies on assuming an $x$-dependence for each of the different PDFs at some starting scale $Q_{0}^{2}$ and then using the Dokshitzer-Gribov-Lipatov-Altarelli-Parisi (DGLAP) evolution equations [16] to model the $Q^{2}$ dependence of the PDFs. This approach is used by both the CTEQ Collaboration [17] and Martin et al. (MRST) [18]; both groups presented progress reports at this workshop. This approach has also been adopted by the ZEUS Collaboration [19], who also presented results of their latest fit at this workshop.

A number of issues have been addressed by both the CTEQ and MRST groups recently. In particular, the compatibility of datasets and the stability of the fit results have been studied.

Both groups have performed studies of fit stability [20, 21], by studying the impact of restricting the fits to data at higher $x$. The studies were performed by looking at the next-to-leading order (NLO) $W^{ \pm}$production cross section predictions from fits with different lower $x$ limits. The results of both studies are shown in figure 8 , The CTEQ group conclude from their studies that the fits are stable. The MRST group conclude that the uncertainties increase significantly as the lower $x$ limit is tightened and that next-to-next-to-leading-order (NNLO) is inherently more stable and these fits should become the standard in the future.

The MRST group also presented the results of other studies, in particular the inclusion of electroweak corrections and QED effects [22]. The latter, in particular, have a negligible effect on the PDFs themselves, as expected. However, the inclusion of QED effects does lead to a small isospin violation, which significantly improves the predictions for prompt photon production at HERA.

The ZEUS Collaboration also presented their latest determination of the proton PDFs using only ZEUS data [23]. In comparison to their previous fits, ZEUS jet cross section data has been included, which is directly sensitive to the gluon density of the proton and benefit from small experimental and theoretical uncertainties. Both NC DIS jet data and direct-enriched dijet photoproduction data, in which the photon behaves as a point-like object, have been included, significantly improving the uncertainty on the gluon PDF in the range $0.01<x<0.4$. If a simultaneous fit of both the PDFs and $\alpha_{s}$ is performed, the 


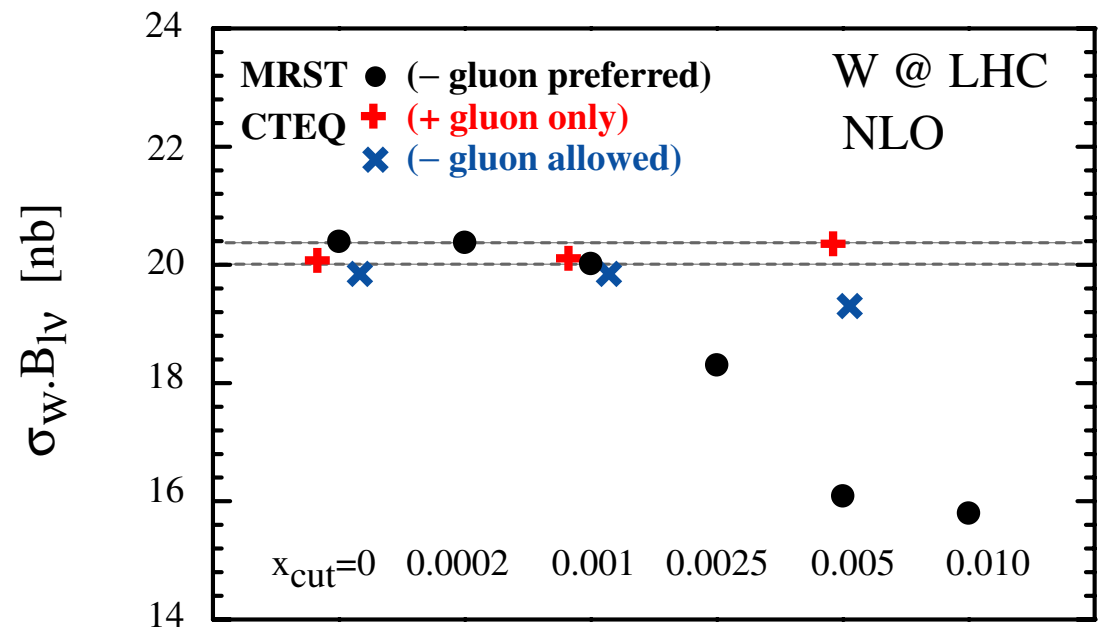

FIGURE 8. Predictions for the $W^{ \pm}$production cross section at the LHC from the CTEQ and MRST Collaborations. The cross sections are plotted as a function of the lower $x$ limit applied to the data used to extract the PDFs. The CTEQ Collaboration have considered two different scenarios: one in which the gluon is forced to be positive-definite and the other in which the gluon is left free. Both are indicated by crosses. The MRST predictions are indicated by the dots and here the gluon is left free.

result for $\alpha_{s}$ is very precise and in agreement with the world average.

Several presentations were also made at this workshop, in which alternative approaches to PDF determination were explained. One such presentation was made by the NNPDF Collaboration [24], who are developing a neural network approach to PDF fitting. This approach avoids some of the shortcomings of the standard method, such as avoiding any potential bias from the choice of functional form for the PDFs. It should also lead to a better estimation of the PDF uncertainties. So far the structure functions have been successfully determined using this approach, but work is still in progress to successfully determine the PDFs using this method.

The estimation and reduction of PDF uncertainties was a key theme at this workshop. One presentation made at this workshop looked at the possibility of averaging the $F_{2}$ data from the H1 and ZEUS experiments, prior to including it in any global PDF fit [25]. This has advantages when it comes to the handling of the systematic uncertainties; it also provides a model-independent method of checking the consistency of the data from the two experiments. It has been found that several contributions to the systematic uncertainties from each experiment are reduced; the experiments are effectively constraining each other. This is an interesting approach which, it is hoped, will be pursued further by the two Collaborations.

Another presentation made at this workshop looked at the impact of future HERA data on the PDF uncertainties [26]. This study was performed using the ZEUS PDF fit as a basis. A number of different scenarios were considered, including simply the expected increase in the amount of luminosity, as well as the inclusion of new cross section measurements which have been optimized to constrain the PDFs as tightly as possible. These improvements would lead to significant improvements in the valence quark distributions, as well as in the high $x$ sea quark and gluon distributions. Other 
scenarios which were also considered are the inclusion of precision measurements of $F_{L}$ from HERA data (low-energy proton running) and the possibility of $e-D$ running to constrain the sea quark asymmetries.

\section{TOWARD QCD PRECISION TESTS}

New HERA data on unpolarized DIS structure functions, combined with the present world data, allow to reduce the experimental error on the strong coupling constant, $\alpha_{S}\left(M_{Z}^{2}\right)$, the fundamental constant of QCD and strong interaction, to the level of $1 \%$. On the theoretial side, the next-to-leading order (NLO) analyses have limitations due to scale variations being present which allow no better than $5 \%$ accuracy in the determination of $\alpha_{s}$ [27]. In order to match the experimental accuracy, it was stressed [27] that analyses of DIS structure functions need to be carried out at the NNLO level. With the recent computation of the 3-loop anomalous dimensions [28], a complete NNLO study of DIS structure functions is now possible. A full NNLO analysis of unpolarized DIS structure functions aiming to obtain a high accuracy determination of $\alpha_{s}$ was presented at the workshop [27]. It was pointed out that a combination of standard NNLO QCD analysis and fits based on factorization scheme-invariant evolution of DIS structure functions will provide a valuable tool in high-precision analyses aiming at $1 \%$ accuracy in the determination of $\alpha_{s}$ [27]. The factorization scheme-invariant evolution of DIS structure functions can be implemented to different pair of structure functions, such as $F_{2}$ and $F_{L}$ or $F_{2}$ and its $t \propto \ln \left(\alpha_{s}(Q)\right)$ derivative. In this approach, $\alpha_{s}$ is determined by performing an one dimensional fit between the evolution of DIS structure functions and the data. Work is still ongoing. A full NNLO accuracy evolution for $F_{2}$ and $\partial F_{2} / \partial t$ have been completely implemented for massless flavors. Inclusion of heavy flavors and the fit to the data, and the one parameter fit to determine $\Lambda_{\mathrm{QCD}}$ are on the way. One interesting result is that comparing the behavior of slopes of $\partial F_{2} / \partial t$ to the slopes extracted experimentally points toward a positive gluon density in small- $x$ region [27], while NLO global analysis of PDFs points to a negative gluon density in low- $x$ and low $Q^{2}$ region [18].

An effort to develop a next generation of event generators was reported at the workshop [29]. The effort was aiming to set up a systematic scheme for developing event generators that are consistent to QCD factorization of differential cross sections up to NLO accuracy. It was argued that in order to achieve this accuracy, one has to use unintegrated PDFs to replace the parton shower in the LO event generators. The basic rules have been established for a DIS event generator, but, there are still works to be done [29].

At hadron colliders, it is the PDFs that determine partonic flux of hard collisions. Full discovery potentials of the LHC and precision tests of QCD are sensitive to PDFs at large $x$. In the form of QCD factorization, extraction of PDFs depends on short-distance dynamics and perturbatively calculated coefficient functions. The coefficient functions often have high powers of logarithms like $\ln (1-x)$ and $\ln (1 / x)$, which become large as $x$ near 1 and 0 . Resummation of these large logarithms is necessary for observables dominated by those kinematic regions. A presentation made at this workshop looked at the effect of large- $x$ resummation on the extraction of PDFs [30]. Large- $x$ resummation was 
performed for coefficient functions of DIS structure functions in massless approximation as well as in an approach that includes heavy quark-mass effects. After performing fits to the fixed target DIS data from NuTeV, BCDMS and NMC collaborations, using NLO and NLL-resummed coefficient functions, it was found that the resummation has a visible impact on the extraction of quark distributions at large $x$, and was stressed that large- $x$ partonic resummation is needed whenever a high precision is required for cross sections evaluated near partonic threshold [30].

A precise knowledge of PDFs, in particular, gluon distribution at $x \sim 0.001-0.01$ are vital for understanding almost all standard production processes at the LHC. When we move away from zero rapidity, much smaller $x$ partons, as small as $10^{-5}$, are required for some observables. Although perturbative QCD has been very successful in interpreting data on scaling violation of PDFs in terms of DGLAP evolution in $Q^{2}$ [17, 18], the extrapolation of PDFs to smaller $x$ has not been very consistent with the BFKL evolution in $x$ (or in energy) [31]. Although the strong rise of proton structure function $F_{2}$ with energy, observed at HERA, can be well described by a simple (3 parameters) LO BFKL fit [32], a much too small effective $\alpha_{s}<0.1$ is needed while the world average is $\alpha_{s} \sim 0.2$ for HERA kinematics. A phenomenological study of confronting NLO BFKL with new HERA data on $F_{2}$ structure function was presented at the workshop [33]. A big discrepancy between theory and data, especially at low $Q^{2}$, was clearly evident [33]. A further study is needed although more progresses have been made recently [34].

\section{LOW- $X$ PHYSICS: PARTON EVOLUTION AND SATURATION}

One of the challenging problems in QCD is to understand the behavior of hadronic cross sections in high energy limit. Experimental data on the total cross section show a slow but distinct rise with collision energy $\sqrt{s}$. This rise could be parametrized by a power of $s, \sigma(s) \sim s^{0.08}$, which is consistent with an exchange of soft Pomerons [35]. On the other hand, after resumming leading powers of $\ln (s)$ contributions, perturbative QCD calculation, in the form of BFKL evolution in energy (or in $x$ ), predicts a much stronger rise with a much large power of $s$ [31]. As $\sqrt{s} \rightarrow \infty($ or $x \rightarrow 0)$, the power-like rise is not compatible with the unitarity of the S-matrix in the high energy limit, or in contradiction with the Froissart bound [36], which allows at most a logarithmic increase with collision energy.

BFKL equation is a linear evolution equation and predicts a large number of low- $x$ partons due to the strength of soft gluon radiation in QCD. On the other hand, the large number of soft partons generated by parton radiation are likely to interact and recombine. Parton recombination introduces non-linear terms into the BFKL equation, slows down the small- $x$ evolution, and removes the apparent violation of the unitarity. When parton recombination is strong enough to balance parton radiation, PDFs saturate as $x \rightarrow 0$ [37]. The state of saturated partons is sometime referred as the Color Glass Condensate (CGC) [38, 39, 40]. A lot of work, both theoretical and experimental, have been done and many progresses have been made in recent years to understand this saturation phenomenon, especially, in a nuclear environment because of an $A^{1 / 3}$ length enhancement in parton density at a given impact parameter. Two sessions at this workshop were devoted to the presentations related to this novel phenomenon. 
A simple modification to the BFKL equation is Balitsky-Kovchekov (BK) equation [41], which adds a quadratic term to the BFKL equation. The BK equation is a nonlinear integro-differential equation for unintegrated PDFs. Its non-linearity leads to many interesting features that could be seen in high energy reactions. It was shown [42] that the BK equation is in the equivalence class of the Fisher-Kolmogorov-PetrovskyPiscounov (FKPP) non-linear partial differential equation, which has so-called traveling wave solutions. The similarity leads to an interesting point of view that high energy QCD is equivalent to a reaction-diffusion system [43]. A detailed numerical studies of the mean field approximation to the BK equation was presented at the workshop [43]. It was demonstrated that the numerical solutions of the BK equation does show features of traveling wave solutions. It was also confirmed that the influence of the initial condition disappears for large $Y \sim \ln (1 / x)$, so that a universal propagation speed is approached, which should help establish statistical interpretations of the phenomena observed in QCD scattering at high energy.

A study of discrete version of the BK equation was presented at this workshop [44]. By noting that the number of gluons in the hadron wave functions is discrete, and their formation in the chain of small $x$ evolution occurs in the discrete intervals of $\ln (1 / x)$, a discrete version of BK equation was formulated [44]. It was found that numerical solutions of the discrete BK equation behave chaotically in the phenomenologically interesting kinematic region. It was concluded [44] that the evolution of the scattering amplitude at high energies in the saturation region might be chaotic, while the scattering amplitude in the normal perturbative region is not affected by the discretization. Although the model used in the numerical calculations neglected the diffusion in transverse momentum, stochasticity of gluon emission and the dynamical fluctuations beyond the mean field approximation, it was hoped that at least some of the features of discrete quantum evolution at small $x$ will survive a more realistic treatment. The chaotic features of small $x$ evolution open a new intriguing prospective on the studies of hadron and nuclear interactions at high energies.

Recent developments of CGC theory were reported at the workshop [45, 46]. The CGC theory is an effective theory of the strong interactions at very high energies [40]. The basic equation of CGC theory is the JIMWLK equation [39], which governs the evolution of a weight function of a color medium (or a hadron target) with rapidity. The evolution kernel is often referred as the JIMWLK Hamiltonian. The weight function is needed for calculating physical scattering amplitude when it is averaged over the medium's color charge. In the large $N_{c}$ limit and in the dipole scattering picture, the JIMWLK equation reduces to the closed and relatively simple BK equation. In a language of Feynman diagrams, the JIMWLK equation includes both BFKL ladder diagrams and the fan diagrams of triple ladder interactions, and it naturally describes the physics of scattering on a dense medium (or a target) with multiple scattering corrections. It naturally interprets the geometric scaling observed in the data [40]. However, the JIMWLK equation does not include Pomeron (or ladder) splittings or all the Pomeron loops. Modifications and improvements to the JIMWLK equation were proposed [45, 46]. In addition, a similar evolution equation was derived for a dilute target [46], while the JIMWLK equation is suitable for scatterings on a dense target. A striking result is that the evolution kernels of these two equations are apparently dual to each other [45, 46]. The selfduality of the kernel is somewhat similar (although different 


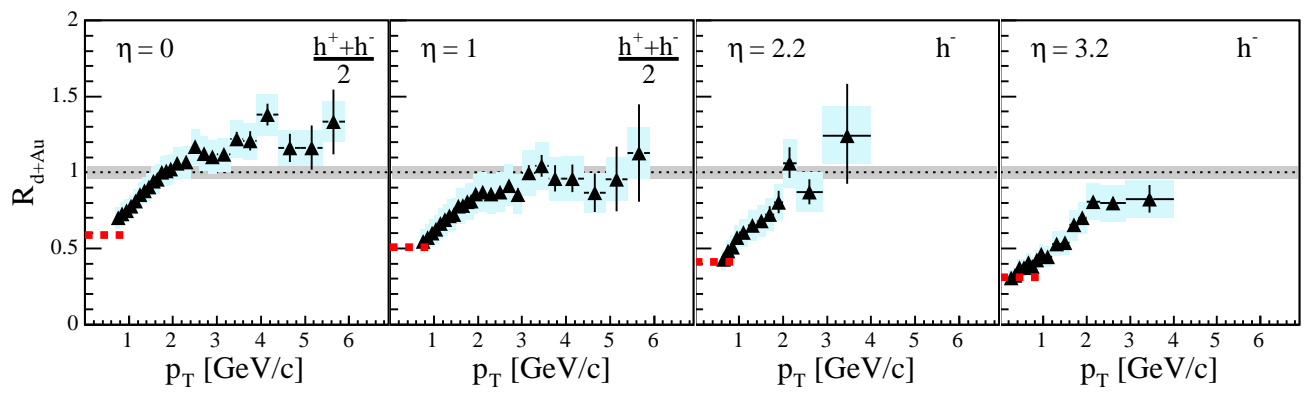

FIGURE 9. Nuclear modification factor for charged hadrons at pseudo-rapidities $\eta=0,1.0,2.2,3.2$. Statistical errors are shown with error bars. Systematic errors are shown with shaded boxes with widths set by the bin sizes. The shaded band around unity indicates the estimated error on the normalization to $\left\langle N_{\text {coll }}\right\rangle$. Dashed lines at $p_{T}<1 \mathrm{GeV} / \mathrm{c}$ show the normalized charged particle density ratio $\frac{1}{\left\langle N_{\text {coll }}\right\rangle} \frac{d N / d \eta(d A u)}{d N / d \eta(p p)}$

in detail) to the duality symmetry, $p \rightarrow x, x \rightarrow-p$ in the Hamiltonian of a harmonic oscillator [46].

Relativistic Heavy Ion Collider (RHIC) is a unique place to test the theory of CGC because of the high density of partons involved. In order to probe small $x$ partons and the phenomena of CGC at RHIC, one has to go to extremely forward and backward region in rapidity because of the relatively low colliding energy. Three major experimental collaborations, BRAHMS, PHENIX, and STAR, at RHIC carried out the effort and presented their early results at the workshop [47, 48, 49].

Rapidity dependence of high- $p_{T}$ particle suppression was measured in d-Au collisions at $\sqrt{s_{N N}}=200 \mathrm{GeV}$ by BRAHMS Collaboration and presented at the workshop [47]. The data collected from d-Au collisions at RHIC is compared to p-p in Figure 9 using the nuclear modification factor defined as

$$
R_{\mathrm{d}-\mathrm{Au}}=\frac{1}{N_{\text {coll }}} \frac{\frac{d N^{\mathrm{dAu}}}{d p_{T} d \eta}}{\frac{d N^{\mathrm{pp}}}{d p_{T} d \eta}}
$$

where $N_{\text {coll }}$ is the number of binary collisions estimated to be $7.2 \pm 0.6$ for minimum biased $\mathrm{d}+\mathrm{Au}$ collisions. Nuclear modification factors for charged hadrons at pseudorapidities $\eta=0,1.0,2.2$ and 3.2 were shown as a function of hadron transverse momentum $p_{T}$. At the central region, or zero rapidity, data confirm the Cronin type enhancement in large $p_{T}$ region. However, as the pseudo-rapidity increases, the enhancement vanishes, and the modification factor is less than the unity for entire measured $p_{T}$ region. The forward region is, the measurement probes target partons with smaller $x$. The observed rapidity dependence of the suppression, which increases with rapidity, fits naturally into the picture of CGC [50], and can be also interpreted by the recombination model of hadronization [51]. In addition, the suppression is consistent with the perturbative QCD calculation based on resummation of coherent multiple scattering [52].

PHENIX Collaboration reported its measurement of charged hadron production in the same d-Au collisions at RHIC [48]. It covers pseudo-rapidities from -2.0 to -1.4 and 1.4 to 2.2 with the forward coverage overlaps with some of BRAHMS measurements. PHENIX also observes a suppression in hadron yields in d-Au collision relative to binary 


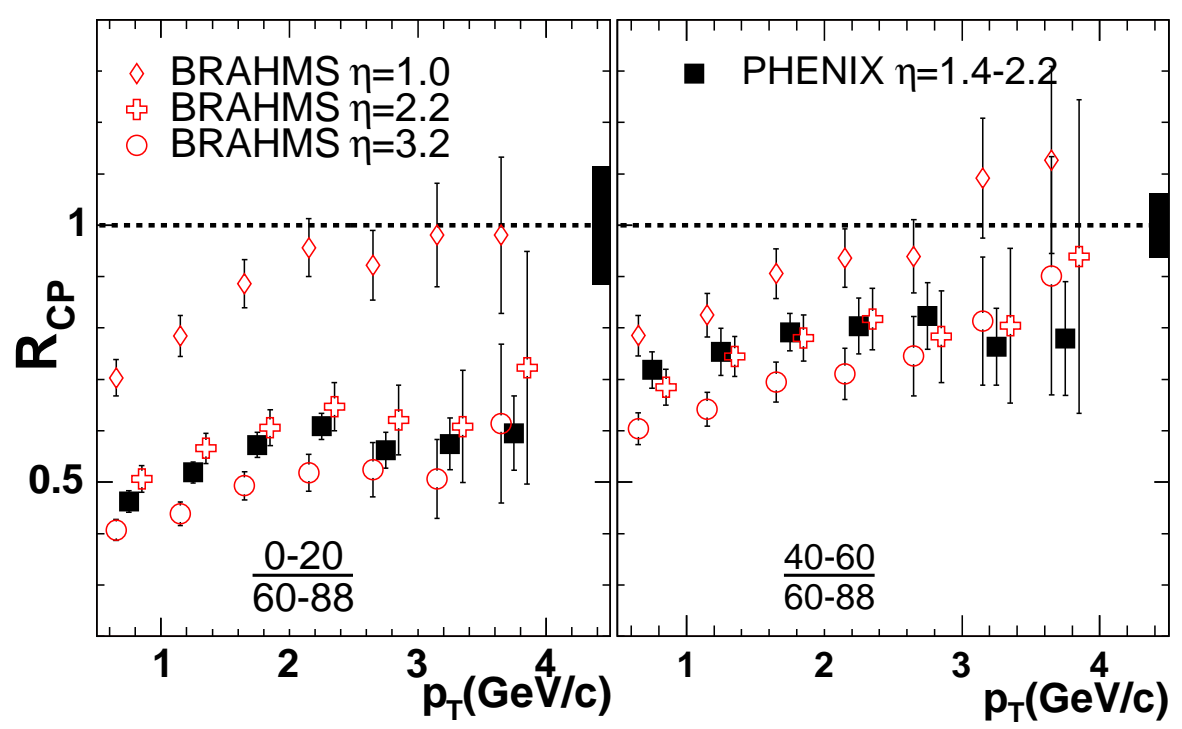

FIGURE 10. PHENIX $R_{c p}$ as a function of $p_{T}$ at forward rapidities shown as the average of the two methods. Note that the BRAHMS results are for negative hadrons at $\eta=2.2,3.2$ and their centrality ranges $(0-20 \% / 60-80 \%$ and $30-50 \% / 60-80 \%)$ are somewhat different from ours.

collision. The data was presented in terms of a different nuclear modification factor, $R_{c p}$, which is defined as the ratio of the particle yield in central collisions to the particle yield in peripheral collisions, each normalized by the averaged number of binary collisions $N_{\text {coll }}$

$$
R_{\mathrm{cp}}=\frac{\frac{d N^{\text {central }}}{d p_{T} d \eta} / N_{\text {coll }}^{\text {central }}}{\frac{d N^{\text {peripheral }}}{d p_{T} d \eta} / N_{\text {coll }}^{\text {peripheral }}} .
$$

As shown in Figure 10, PHENIX data are consistent with BRAHMS data, and are in qualitative agreement with theoretical expectation. Quantitatively, the ratio $R_{c p}$ for the most central over the most peripheral collisions is more suppressed than theoretical calculations.

Measurements of the inclusive yields of $\pi^{0}$ mesons in $\mathrm{p}$-p and $\mathrm{d}$-Au collisions at RHIC were presented by STAR Collaboration [49]. With a forward $\pi^{0}$ detector installed at the Solenoidal Tracker at RHIC (STAR), it can detect high energy $\pi^{0}$ mesons with pseudo-rapidity as large as $3.3<\eta<4.1$ [49]. The inclusive yield in p-p collisions at $\sqrt{s}=200 \mathrm{GeV}$ are consistent with NLO pQCD calculations. The nuclear modification factor, $R_{d A u}$ in Figure 11, shows a strong suppression at the large pseudo-rapidity. It was argued that the d-Au yield is consistent with a model calculation treating the Au nucleus as a CGC for forward particle production [49]. Comparisons with other production models will be interesting to perform. Additional measurements with different finalstate particles and at different centralities will help elucidate the cause of the observed strong suppression, which covers a broad range of nuclear gluon momentum fraction with a peak value $x \sim 0.02$ [49]. 

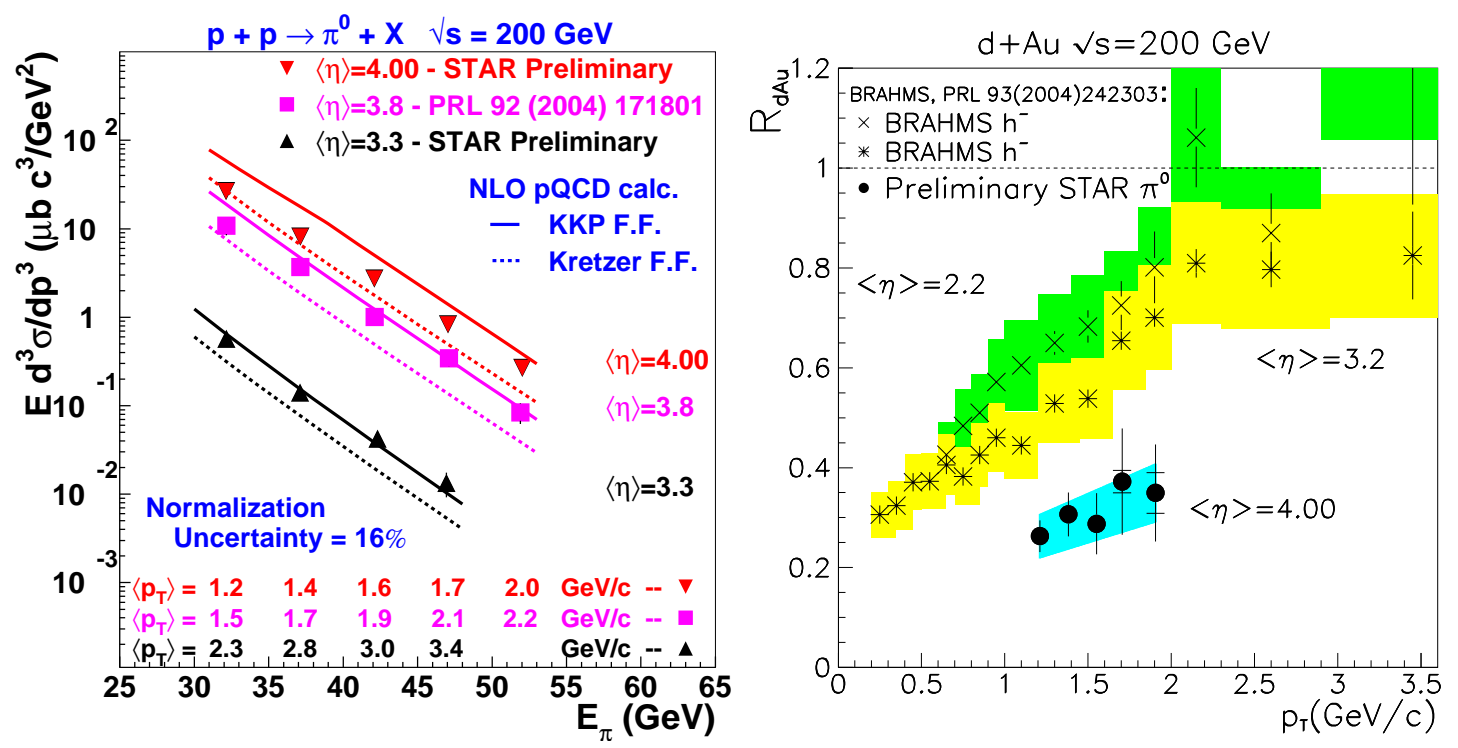

FIGURE 11. Inclusive $\pi^{0}$ yield for $\mathrm{p}+\mathrm{p}$ [left] and $\mathrm{d}+\mathrm{Au}$ collisions normalized by $\mathrm{p}+\mathrm{p}$ [right]. The pion energy $\left(E_{\pi}\right)$ is correlated with the transverse momentum $\left(p_{T}\right)$, as the FPD was at fixed values of pseudo-rapidity $(\eta)$. The inner error bars are statistical, while the outer combine these with the $E_{\pi^{-}}\left(p_{T^{-}}\right.$ ) dependent systematic errors, and are often smaller than the points. The curves (left) are NLO pQCD calculations evaluated at fixed $\eta$, using different fragmentation functions. The x's and stars (right) are BRAHMS data for $h^{-}$production at smaller $\eta$.

Two theory talks were presented at the workshop to specifically address the strong suppression observed in the forward region of d-Au collisions at RHIC [50, 51]. Two completely different pictures were presented on how a leading hadron was produced in the d-Au collisions at RHIC energies. In one approach [50], single hadron production was assumed to be proportional to gluon production. Under this approximation, the nuclear modification factor $R_{d A u}$ is the same for both hadron and gluon production. The gluon production in d-Au collisions was calculated in the framework of CGC physics [50]. In the other approach [51], a single hadron was produced via recombination of partons available during the collisions. It was argued that p-p, p(d)-Au, and Au-Au collisions produce different shapes of parton spectra. Recombination of partons with different spectra naturally leads to different hadron distribution and a nontrivial nuclear modification factor [51]. A striking fact is that both of these approaches provided calculations that are consistent with the observed data.

\section{NUCLEAR STRUCTURE FUNCTIONS AND NUCLEAR PDFS}

It was observed about two decades ago that DIS structure functions of nuclei differ from simple sum of those in the free nucleon [53]. As a result, PDFs of a nucleus of atomic weight $A$ also differ from those in the free proton, $f_{i}^{A}\left(x, Q^{2}\right) \neq f_{i}\left(x, Q^{2}\right)$. In order to understand the overwhelming data from the RHIC and make predictions for the heavy ion programs at the future facilities, like the LHC and Electron Ion Collider (EIC), we need precise information of nuclear PDFs (nPDFs), in particular, at small $x(<0.1)$. 
A brief overview of the global DGLAP analyses of nPDFs was presented at the workshop [54]. The nPDFs are defined in terms of the same operators that define the free nucleon PDFs with the free nucleon state replaced by a nuclear state. Therefore, nPDFs and free nucleon PDFs should share the same DGLAP evolution equations, and only difference between nPDFs of different nuclei and free nucleon PDFs is the input distributions to DGLAP equations at a scale $Q_{0}^{2}$. Once a set of the nonperturbative input distributions are chosen, DGLAP evolution equations predict nPDFs at a larger momentum scale $Q^{2}$. There are typically two approaches to choose the input distributions: calculated by the nuclear models and determined by fit to the data [54]. The second approach shares the same procedures as that used in the determination of PDFs, and often referred as the global analyses of nPDFs. There are three groups who have been carrying out the global analyses and reanalyses of nPDFs: Eskola et al. (usually called as EKS98) [55, 56, 57], Hirai et al. [58, 59], and de Florian and Sassot [60]. The first two groups use LO DGLAP evolution while the third uses NLO evolution. All analyses, only DIS and Drell-Yan data on nuclear targets were used in the fits. Because of the large error in nuclear data and the lack of direct information on gluon initiated processes, all fits have reasonable constrains and consistencies on quark distributions, but, not on gluon distributions [54].

A hard probe often refers to a scattering process with a large momentum exchange $q^{\mu}$ whose invariant mass $Q \equiv \sqrt{\left|q^{2}\right|} \gg \Lambda_{\mathrm{QCD}}$, and it can probe a distance scale much smaller than size of a nucleon at rest, $1 / Q \ll$ fm. However, when an active parton's momentum fraction $x<x_{c} \sim 0.1$, a hard probe might interact with more than one partons of the nucleon coherently [61]. When $x \ll x_{c}$, the hard probe can cover a whole Lorentz contracted nucleus and interact with partons from different nucleons. Although such coherent multi-parton interactions are power suppressed by hard scales of the scattering, they are enhanced by the nuclear size and could be one of the important sources of nuclear dependence observed in high energy nuclear collisions. A presentation made at this workshop looked at the impact of coherent multiple scattering in DIS on nuclear targets and leading particle production in $\mathrm{p}(\mathrm{d})$-Au collisions [52]. An all power resummation of nuclear enhanced power corrections to DIS structure functions on nuclear targets was achieved. The calculated results for the Bjorken $x$-, $Q^{2}$ - and $A$-dependence of nuclear shadowing in $F_{2}^{A}\left(x, Q^{2}\right)$ and the nuclear modifications to $F_{L}^{A}\left(x, Q^{2}\right)$ are consistent with the existing data [52]. Predictions were made for the dynamical shadowing from final state interactions in $v+A$ reactions for sea and valence quarks in the structure functions $F_{2}^{A}\left(x, Q^{2}\right)$ and $x F_{3}^{A}\left(x, Q^{2}\right)$, respectively. In addition, calculations for the centrality and rapidity dependent nuclear suppression of single and double inclusive hadron production at moderate transverse momenta in $p+A$ collisions were presented and consistent with the RHIC data [52].

In the Gribov-Glauber picture, nuclear shadowing and antishadowing observed in nuclear structure functions are due to the destructive and constructive interference of amplitudes arising from the multiple-scattering of quarks in the nucleus, respectively. A calculation of shadowing and antishadowing of nuclear structure functions in the Gribov-Glauber picture were presented at the workshop [62]. The coherence of multistep nuclear processes leads to shadowing and antishadowing of the electromagnetic nuclear structure functions in agreement with the data. But, the same picture leads to 
substantially different antishadowing for charged and neutral current reactions, thus affecting the extraction of the weak-mixing angle $\theta_{W}$ [62]. This is due to the fact that Reggeon couplings depend on the quantum numbers of the struck quark implies nonuniversality of nuclear antishadowing for charged and neutral currents [62].

\section{NEW APPROACHES TO PDFS}

Moments of PDFs are matrix elements of local gauge invariant operators which in principle can be calculated by using lattice QCD. A brief review of recent lattice effort in determining the PDFs was presented at the workshop [63]. Lattice QCD calculations of three representative observables, the transverse quark distribution, momentum fraction, and axial charge, were presented [63]. It was emphasized that lattice calculations of nucleon structure are beginning to realize their promise to elucidate QCD and make contact with the experimental programs. It was concluded that recent calculations are painting a qualitative three dimensional picture of nucleon structure revealing a significant $x$ dependence of the transverse size of the nucleon. Quantitative calculations of moments of PDFs are progressing, in particular, the calculation of $g_{A}$ may soon reach a few percent accuracy.

An analytical approach to understand the three dimensional picture of nucleon structure was presented at the workshop [64]. A concept of the quantum phase-space (Wigner) distributions for the quarks and gluons in the nucleon was introduced. The quark Wigner functions were related to the transverse-momentum dependent PDFs and generalized PDFs with emphasis on the physical role of the skewness parameter. Any knowledge on the generalized PDFs can be immediately translated into the correlated coordinate and momentum distributions of partons. In particular, the generalized PDFs can be used to visualize the phase-space motion of the quarks, and hence allow studying the contribution of the quark orbital angular momentum to the spin of the nucleon. It was concluded that measurements of generalized PDFs and/or direct lattice QCD calculations of them will provide a fantastic window to the quark and gluon dynamics in the proton [64].

Another presentation made at the workshop looked at quark asymmetries in nucleons [65]. Instead of fitting the data, a physical model for the non-perturbative $x$-shape of PDFs was developed. The model was based on Gaussian fluctuations in momenta, and quantum fluctuations of the proton into meson-baryon pairs. It was found that the model gives a good description of the proton structure function and a natural explanation of observed quark asymmetries, such as the difference between the anti-up and anti-down sea quark distributions and between the up and down valence distributions [65]. Within this model, there is an asymmetry in the momentum distributions of strange and antistrange quarks in the nucleon, and the asymmetry is large enough to reduce the NuTeV anomaly to a level which does not give a significant indication of physics beyond the standard model.

Effective field theory was used to investigate the nuclear modification to the PDFs and a recent result was presented at the workshop [66]. It was found that the universality of the shape distortion in nPDFs (the factorization of the Bjorken $x$ and atomic weight $A$ dependence) is model independent and emerges naturally in effective field theory. For 
a simple parameterization of nonperturbative functions in the approach, fits to the data confirm the factorization [66].

\section{ACKNOWLEDGMENTS}

We would like to thank all the members of our working group for the excellent presentations and for lively discussions they provoked. We would also like to thank the conveners of the Electroweak and Beyond the Standard Model working group for their assistance in the joint session on high $Q^{2}$ structure function measurements. We would also like to thank all the session chairs for agreeing to be involved. Last, but not least, we would like to thank the organizers of DIS 2005 for interesting and well-organized meeting.

\section{REFERENCES}

1. ZEUS Collaboration, S. Chekanov et al. Eur. Phys. J. C21, 443-471 (2001); H1 Collaboration, C. Adloff et al. Eur. Phys. J. C21, 33-61 (2001).

2. ZEUS Collaboration, S. Chekanov et al. Phys. Lett. B487, 1-2, 53-73 (2000).

3. E. Lobodzinska, these proceedings; H1 Collaboration, A. Aktas et al. Phys. Lett. B598, 159-171 (2004).

4. A. Petrukhin, these proceedings.

5. H1 Collaboration, A. Nikiforov, these proceedings; ZEUS Collaboration, A. Tapper, these proceedings.

6. ZEUS Collaboration, Y. Ning, these proceedings.

7. NuTeV Collaboration, M. Tzanov, these proceedings, hep-ex/0507040.

8. S. Kuhlmann et al, Phys. Lett. B476,291 (2000).

9. S. Kuhn, these proceedings.

10. CDF Collaboration, Y.S. Chung, these proceedings.

11. S. Liuti, these proceedings.

12. C. Keppel, these proceedings; I. Niculescu, these proceedings.

13. A. Bodek, these proceedings.

14. H. Gallagher, these proceedings.

15. F. Chlebana, these proceedings; A. Harel, these proceedings.

16. Yu. Dokshitzer, Soviet Phys. JETP 46, 641 (1977); V. N. Gribov and L. N. Lipatov, Soviet J. Nucl. Phys. 15, 438,675, (1972); L. N. Lipatov, Soviet J. Nucl. Phys. 20, 95, (1975); G. Altarelli and G. Parisi, Nucl. Phys B126, 298 (1977).

17. D. Stump, these proceedings.

18. R. Thorne, these proceedings, hep-ph/0507015.

19. J. Terron, these proceedings.

20. J. Huston, J. Pumplin, D. Stump and W. K. Tung, hep-ph/0502080.

21. A. Martin, R. Roberts, W. J. Stirling and R. Thorne, Phys. Lett. B604, 61-68 (2004).

22. A. Martin, R. Roberts, W. J. Stirling and R. Thorne, Eur. Phys. J. C39, 155-161 (2005).

23. ZEUS Collaboration, S. Chekanov et al. DESY 05-050.

24. J. Rojo, these proceedings, hep-ph/0505044.

25. A. Glazov, these proceedings.

26. C. Gwenlan, these proceedings.

27. A. Guffanti, these proceedings.

28. S. Moch, J. A. M. Vermaseren and A. Vogt, Nucl. Phys. B688, 101-134 (2004); B691, 129-181 (2004).

29. X. Zu, these proceedings; J. C. Collins and X. Zu, JHEP 0503, 059 (2005); 0206, 018 (2002).

30. G. Corcella, these proceedings. 
31. L. N. Lipatov, Sov. J. Nucl. Phys. 23, 338 (1976); E. A. Kuraev, L. N. Lipatov and V. S. Fadin, Sov. Phys. JETP 45, 199-204 (1977); I. I. Balitsky and L. N. Lipatov, Sov. J. Nucl. Phys. 28, 822-829 (1978).

32. H. Navelet, R. Peschanski, C. Royon, and S. Wallon, Phys. Lett. B385, 357-364 (1996); S. Munier and R. Peschanski, Nucl. Phys. B524, 377-393 (1998).

33. C. Royon, these proceedings.

34. G. Altarelli, R. D. Ball and S. Forte, Nucl. Phys. Proc. Suppl. 135, 163-167 (2004).

35. A. Donnachie, P.V. Landshoff, Phys. Lett. B296, 227 (1992).

36. M. Froissart, Phys. Rev. 123, 1053 (1961).

37. L. V. Gribov, E. M. Levin and M. G. Ryskin, Phys. Rept. 100, 1-150 (1983); A. H. Mueller and J. W. Qiu, Nucl. Phys. B268, 427 (1986); A. H. Mueller, Nucl. Phys. B558, 285 (1999).

38. L. D. McLerran and R. Venugopalan, Phys. Rev. D49, 3352 (1994); Phys. Rev. D49, 2233 (1994); Phys. Rev. D50, 2225 (1994).

39. J. Jalilian-Marian, A. Kovner, A. Leonidov and H. Weigert, Phys. Rev. D59, 014014 (1999); J. JalilianMarian, A. Kovner and H. Weigert, Phys. Rev. D59, 014015 (1999).

40. E. Iancu, A. Leonidov and L. D. McLerran, Nucl. Phys. A692, 583 (2001); Phys. Lett. B510, 133 (2001); E. Iancu and L. D. McLerran, Phys. Lett. B510, 145 (2001).

41. I. Balitsky, Nucl. Phys. B463, 99 (1996); Phys. Rev. Lett. 81, 2024 (1998); Phys. Lett. B518, 235 (2001); Y.V. Kovchegov, Phys. Rev. D60, 034008 (1999); Phys. Rev. D61, 074018 (2000).

42. S. Munier and R. Peschanski, Phys. Rev. Lett. 91, 232001 (2003); Phys. Rev. D69, 034008 (2004); Phys. Rev. 70, 077503 (2004).

43. R. Enberg, these proceedings.

44. K. Tuchin, these proceedings.

45. A. M. Stasto, these proceedings.

46. A. Kovner, these proceedings.

47. F. Videbaek for BRAHMS Collaboration, these proceedings.

48. C. Zhang for PHENIX Collaboration, these proceedings.

49. G. Rakness for STAR Collaboration, these proceedings.

50. Y. Kovchegov, these proceedings.

51. R. Fries, these proceedings.

52. I. Vitev, these proceedings.

53. J. Aubert et al., Phys. Lett. B123, 275 (1983).

54. V. Kolhinen, these proceedings.

55. K. J. Eskola, V. J. Kolhinen, and P. V. Ruuskanen, Nucl. Phys. B535, 351-371 (1998).

56. K. J. Eskola, V. J. Kolhinen, and C. A. Salgado, Eur. Phys. J. C9, 61-68 (1999).

57. K. J. Eskola, V. J. Kolhinen, and C. A. Salgado, In preparation.

58. M. Hirai, S. Kumano, and M. Miyama, Phys. Rev. D64, 034003 (2001).

59. M. Hirai, S. Kumano, and T. H. Nagai, Phys. Rev. C70, 044905 (2004).

60. D. de Florian, and R. Sassot, Phys. Rev. D69, 074028 (2004).

61. J. W. Qiu, Nucl. Phys. A 715, 309 (2003) arXiv:nucl-th/0211086.

62. S.J. Brodsky, these proceedings.

63. D. Renner, these proceedings.

64. A. V. Belitsky, these proceedings; A. V. Belitsky, X. Ji and F. Yuan, Phys.Rev. D69, 074014 (2004).

65. J. Alwall, these proceedings.

66. W. Detmold, these proceedings. 